\title{
PREFERENCIA POR MORFOESPECIES DE BABOSAS EN DIFERENTES CULTIVOS Y AMBIENTES DEL MUNICIPIO PAMPLONA, NORTE DE SANTANDER
}

\section{PREFERENCE BY MORPHO SPECIES OF SLUGS IN DIFFERENT CROPS AND ENVIRONMENTS IN THE PAMPLONA MUNICIPALITY, NORTE DE SANTANDER.}

\author{
Leónides Castellanos González¹, Junior Serrano Ortiz², WIda M. \\ Becerra Rozo
}

\section{Entidad}

${ }^{1}$ PhD. Facultad de Ciencias Agrarias. Universidad de Pamplona. Km 1 vía Bucaramanga E-mail: Iclcastell@gmail.com, ORCID: http://orcid.org/0000-0001-9285-4879

2 Ingeniero agrónomo. Facultad de Ciencias Agrarias. Universidad de Pamplona. Km 1 vía Bucaramanga E-mail: jakson1@outlook.es

${ }^{3}$ MSc. Facultad de Ciencias Agrarias. Universidad de Pamplona. Km 1 vía Bucaramanga E-mail wmargi26@gmail.com

\section{Resumen}

El trabajo tuvo como objetivo evaluar la preferencia y el perjuicio causado por morfoespecies de babosas en diferentes cultivos en el municipio Pamplona, Norte de Santander. La investigación se desarrolló en cinco veredas de Pamplona Norte de Santander de septiembre a diciembre de 2018. Se evaluaron nueve cultivos, arveja, cebolla de bulbo, fresa, frijol, papa criolla, papa negra, tomate de árbol y zanahoria, dando un total de cincuenta campos. Se realizó la identificación de las morfoespecies por las características morfológicas mediante estereoscopio basado en una clave taxonómica. Se identificaron cinco morfoespecies de babosas afectando los cultivos pertenecientes a las familias Agriolimacidae, Arionidae y Milacidae. Arion sp. ${ }^{1}$, Deroceras sp. ${ }^{1}$ y Deoceras $\mathrm{sp.}^{2}$ estaban presentes en las cinco veredas; Deroceras sp. ${ }^{3}$ no se encontró en la vereda Chíchira mientras que Milax sp. ${ }^{1}$ solo se consiguió en las veredas El Rosal, El Totumo y Monteadentro, prefiriendo todas las morfoespecies al cultivo de fresa y tomate de árbol. La incidencia de daño por los moluscos se evidencia en los nueve cultivos muestreados, sin embargo, la mayor preferencia se observa en papa negra, papa criolla, zanahoria y alverja con los valores más altos de incidencia y severidad, resaltándose el alto nivel de daño ocasionado en los frutos del cultivo de fresa.

Palabras clave: Moluscos, Daño, Fresa, Papa, Arveja, Zanahoria, Tomate De Arbol 


\begin{abstract}
The objective of the work was to evaluate the preference and the damage caused by morpho-species of slugs in different crops in the municipality Pamplona, Norte de Santander. The research was carried out in five paths of Pamplona Norte de Santander from September to December 2018. Nine crops, peas, bulb onion, strawberry, beans, Creole potatoes, black potatoes, tree tomatoes and carrots were evaluated, giving a total of Fifty fields Morphospecies were identified by morphological characteristics using a stereoscope based on a taxonomic key. Five morpho-species of slugs were identified affecting the crops belonging to the Agriolimacidae, Arionidae and Milacidae families. Arion sp.1, Deroceras sp.1 and Deoceras sp.2 were present in the five paths; Deroceras sp.3 was not found on the Chíchira path while Milax sp. 1 was only found on the El Rosal, El Totumo and Monteadentro paths s. All morphospecies preferred to strawberry and tree tomato cultivation. The incidence of damage from mollusks is evident in the nine sampled crops, however, the highest preference is observed in black potatoes, creole potatoes, carrots and peas with the highest incidence and severity values, highlighting the high level of damage caused in the fruits of the strawberry crop.

Key words: Mollusks, Damage, Strawberry, Potato, Pea, Carrot, Tree Tomato
\end{abstract}

\section{INTRODUCCIÓN}

Las babosas constituyen plagas de gran importancia agrícola (Monje, 1996). Estas pueden desarrollarse entre un amplio rango de temperatura (Cañedo, Alfaro \& Kroschel, 2011); teniendo gran preferencia para hacer actividad por los climas templados (Serre, 2005) y tienen gran preferencia por humedades relativas altas (Herrera \& Castellanos, 2013; Villamizar \& Justinico, 2017).

En sur América se reportan daños por babosas en países que cumplen con las condiciones ya mencionadas como es el caso en Perú, reportando a las especies Agriolimax spp., Limax spp. y Vaginulus spp., ocasionando daños en gran variedad de cultivos (Cañedo, Alfaro \& Kroschel, 2011; Velandia et al, 2016).

En Colombia se encuentran moluscos plagas en pisos térmicos que presenten humedades relativas superiores al $80 \%$. Según el ICA (2012), se menciona que, los moluscos causan daño al follaje, tubérculos y raíces de las plantas, con una amplia variedad de cultivos atacados.
Uno de los casos más importantes se da en el cultivo del café, ocasionando lesiones en los frutos hasta la caída de los mismos, raspadura de tallos, marchitamiento total de almácigos por anillado del tallo, entre otros perjuicios (Constantino, Gomes \& Benavides, 2010).

Entre otros productos agrícolas de Colombia, se tienen las hortalizas entre ellas repollo, coliflor, lechuga, espinacas y acelgas, reportando perjuicios por Deroceras reticulatum Muller, 1774, Limax marginatus Muller, 1774 y Milax gagates Draparnaut, 1801 (ICA, 2012); estas especies también coinciden con los moluscos a los cuales se les atribuyen los perjuicios posibles en los cultivos de papa negra y criolla. En el caso de frutales anuales de consumo en fresco como el cultivo de mora, se atribuyen las afecciones presentes a Milax gagates Draparnaut (ICA, 2011.a).

El municipio de Pamplona, cuenta con una remarcada inclinación de la producción agrícola por los productos hortofrutícolas, al contar con las condiciones agroecológicas adecuadas tanto para la producción de frutas y hortalizas, como para los moluscos que pueden ser 
hospederos de una gran variedad de cultivos. Reconociendo la importancia del cultivo de fresa, siendo uno de los productos relevantes en la explotación agrícola presente en la económica de Pamplona (Gualdron, Maldonado, Espitia \& Garcia, 2017). Las babosas, son una plaga en común en la horticultura y en el cultivo de fresa, gracias a la amplia gama de especies vegetales que puede hacer su hospedero. Esta plaga, se ve favorecida por las condiciones meteorologías presentes de la zona, como lo son la humedad relativa alta, constante precipitación, temperaturas entre los $5^{\circ} \mathrm{C}$ los $25^{\circ} \mathrm{C}$, entre otras, necesarias para la proliferación de su especie (Serre, 2005).

Aunque en las condiciones de Pamplona, Norte de Santander, gran parte de los cultivos presentan daños a causa de las babosas, el estudio de estos pulmonados para el municipio se resume a los de Hernández, Guerrero y Sierra (2015), sobre la especie de babosa Arion distinctus (Mabille) que abunda en la región, a los de Méndez y Castellanos (2017) que evaluaron la cal agrícola y la tierra de diatomeas contra el caracol del jardín Helix aspersa Muller en condiciones de laboratorio y a los de Méndez y Castellanos (2019) que evaluaron esos productos alternativos contra Arionidos y Agrolimaceos en condiciones de laboratorio y campo. Teniendo en cuenta estos antecedentes el objetivo de la investigación fue evaluar la preferencia y el perjuicio causado por morfoespecies de babosas en diferentes ambientes $y$ cultivos en el municipio Pamplona, Norte de Santander.

\section{METODOLOGIA}

La investigación se desarrolló en cinco de los cinco ambientes (veredas agrícolas) del municipio Pamplona Norte de Santander: Chíchira, El Escorial, El Rosal El Totumo y Monteadentro de septiembre a diciembre de 2018. En la vereda
Chíchira se ingresó en cinco (5) predios, en El Escorial a cuatro (4) predios y en EI Rosal se muestrearon seis (6) predios. De igual manera en las veredas El Totumo se ingresó a cinco (5) predios y en Monteadentro a seis (6). En cada predio se tomaron muestras de especímenes de babosas, para su posterior identificación, así como para la cuantificación de los perjuicios empleando una escala de la intensidad del daño por los moluscos en los cultivos presentes.

\section{Morfoespecies de babosas presentes en los cultivos.}

Para la toma de muestra de los moluscos presentes en cada cultivo se emplearon recipientes con alcohol etílico al $70 \%$ para la conservación de especímenes muertos y recipientes con sustrato del cultivo en el cual eran encontrados. Estos se buscaron mediante observación del rastro de los individuos para la recolección, priorizando la búsqueda cerca de las zonas no cultivadas, entre pastizales, bajo piedras y terrones dentro del cultivo.

Los especímenes recolectados en campo eran llevados al laboratorio de Biología de la Facultad de Ciencias Básicas de la Universidad de Pamplona para la identificación mediante claves taxonómicas. La identificación se realizó por las características morfológicas mediante estereoscopio basado en una clave taxonómica (Harwood, Thomas, Mc Donnell \& Paine, 2010), lo que permitió llegar hasta nivel de familia y género las morfoespecies de babosas encontradas. Finalizada la fase de identificación se construyó un conglomerado mediante Excel para el procesamiento de la información y la relación de los especímenes con la preferencia por cultivos.

Perjuicios por babosas en los cultivos. Simultaneo a la recolección de muestras de ejemplares de babosas, se ejecutó la cuantificación de los perjuicios mediante la aplicación de una escala de intensidad de 
daño por parte de las babosas en cada cultivo, dando un valor de porcentaje de incidencia y severidad de daño. Los cultivos encontrados y monitoreados en campo mediante las visitas fueron nueve (9), arveja, cebolla de bulbo, fresa, frijol, papa criolla, papa negra, tomate de árbol y zanahoria, dando un total de cincuenta (50) campos.

Para el monitoreo y la búsqueda de las babosas se tuvieron en cuenta los siguientes criterios: Concentrar los muestreos en lugares cercanos a arboledas, sectores enmalezados, en las proximidades de los bordes de arroyos, bordes de los campos, priorizando las medias lomas (Serre, 2005).

Los sitios de muestreo estuvieron distribuidos dentro y en los márgenes del cultivo siguiendo un patrón $\mathrm{W}$ para determinar si todo el cultivo o solo una fracción del mismo, estaba con la presencia de las babosas. Se monitorearon cincuenta (50) sitios como mínimo, por lote para tener una buena estimación de la naturaleza y extensión de la infestación de babosas (Serre, 2005).

Dependiendo de la especie cultivada se tuvo en cuenta la escala de evaluación asociada al grado de afectación de las plantas, tomando 50 plantas por cultivo, calificando mediante observación el grado de afección por babosa en cada planta, basado en estalas de grado de afectación ya establecidas (Tabla 1):

Tabla 1. Escala de grados de daño empleada para la evaluación en campo.

\begin{tabular}{lll}
\hline $\begin{array}{l}\text { Grado } \\
\text { afectación }\end{array}$ & $\begin{array}{l}\text { de } \\
\text { Porcentaje } \\
\text { afectación } \\
\text { observado (\%) }\end{array}$ \\
\hline 0 & $\begin{array}{l}\text { Sin afectación } \\
\text { Menor o igual a 5 } \\
1\end{array}$ & Desde 6 hasta 25 \\
2 & Desde 26 hasta 50 \\
3 & Desde 51 hasta 75 \\
4 & Mayor a 75 \\
5
\end{tabular}

Nota: Tomado del Manual de ensayos de campo en protección vegetal por Ciba-Geigy (1981).

En el caso de la fresa al presentar solo daño en los frutos se optó por monitoreo de 100 frutos por todo el predio, calificando la presencia o ausencia de daño por moluscos, determinando solo la incidencia. Basado en estos datos se calculó la incidencia y severidad en cada caso, exceptuando la fresa.

Para el cálculo de la incidencia se tomó la existencia de plantas $u$ órganos afectados respecto al total evaluado.

$$
\% \text { Incidencia }=\frac{\text { plantas u organos afectados }}{\text { plantas u organos evaluados }} * 100
$$

Para la severidad se tuvo en cuenta de la especie de planta evaluada, es decir se puede asignar un grado de perjuicio (Griffiths, Phillips, Compton, Wright \& Incoll, 1998).

$$
\% \text { Sever. }=\frac{\sum(\text { grado } * \text { plantas en ese grado })}{\text { grado máximo } * \text { plantas evaluadas }} * 100
$$

Basado en los datos obtenidos se calculó la incidencia y severidad ponderada para cada vereda, en cada especie cultivada.

Donde:

$$
\text { Incidencia ponderada }=\frac{\Sigma A_{i} * I_{i}}{\Sigma A_{i}}
$$

$\mathbf{A}_{\mathbf{i}}=$ área de incidencia en cada campo o unidad de muestreo.

$\mathbf{I}_{\mathrm{i}}=$ índice de incidencia en cada campo o unidad de muestreo.

Donde:

$$
\text { Severidad ponderada }=\frac{\Sigma A_{i} * S_{i}}{\Sigma A_{i}}
$$

$\mathbf{A}_{\mathrm{i}}=$ área de incidencia en cada campo o unidad de muestreo.

$\mathbf{S}_{\mathrm{i}}=$ índice de incidencia en cada campo o unidad de muestreo.

\section{ANALISIS $Y$ DISCUSIÓN DE RESULTADOS}




\section{Especies de babosas presentes en los cultivos.}

De la Familia Agriolimacidae se observaron tres (3) morfoespecies pertenecientes al género Deroceras, se caracterizaban por presentar pneumostoma posicionado en la zona posterior del manto, quilla corta o poco visible y una pequeña membrana rodeando la quilla.

La primera morfoespecie de este género encontrada en las visitas a campo, evidenciaba un tamaño de $2,5 \mathrm{~cm}$ aproximadamente, un color castaño, moteada o con serie de "pecas" sobre el cuerpo y un manto con surcos en forma de espiral. Dicho espécimen se encontró en los cultivos de zanahoria, fresa, arveja, papa criolla, papa negra, tomate de árbol, mora y frijol, por lo cual se podría suponer su preferencia por estos cultivos, al igual que los otros especímenes encontrados en cada cultivo. Este se nombró, Deroceras sp. ${ }^{1}$ (Figura 1).

La segunda morfoespecie determinada nombrada Deroceras sp. ${ }^{2}$, con un tamaño de $2,5 \mathrm{~cm}$ aproximadamente, estaba caracterizada por presentar un color anaranjado y un manto relativamente liso, tuvo preferencia por los cultivos de zanahoria, fresa, mora y tomate de árbol. La última morfoespecie encontrada nombrada Deroceras sp. ${ }^{3}$, presentaba un tamaño de 3,0 cm aproximadamente, color miel un poco similar a Deroceras sp. ${ }^{1}$, sin embargo, su manto espiralado ni su cuerpo tenian "pecas" . Los especímenes pertenecientes a esta morfoespecie fueron encontrados en cultivos de zanahoria, fresa, arveja, cebolla bulbo y frijol.

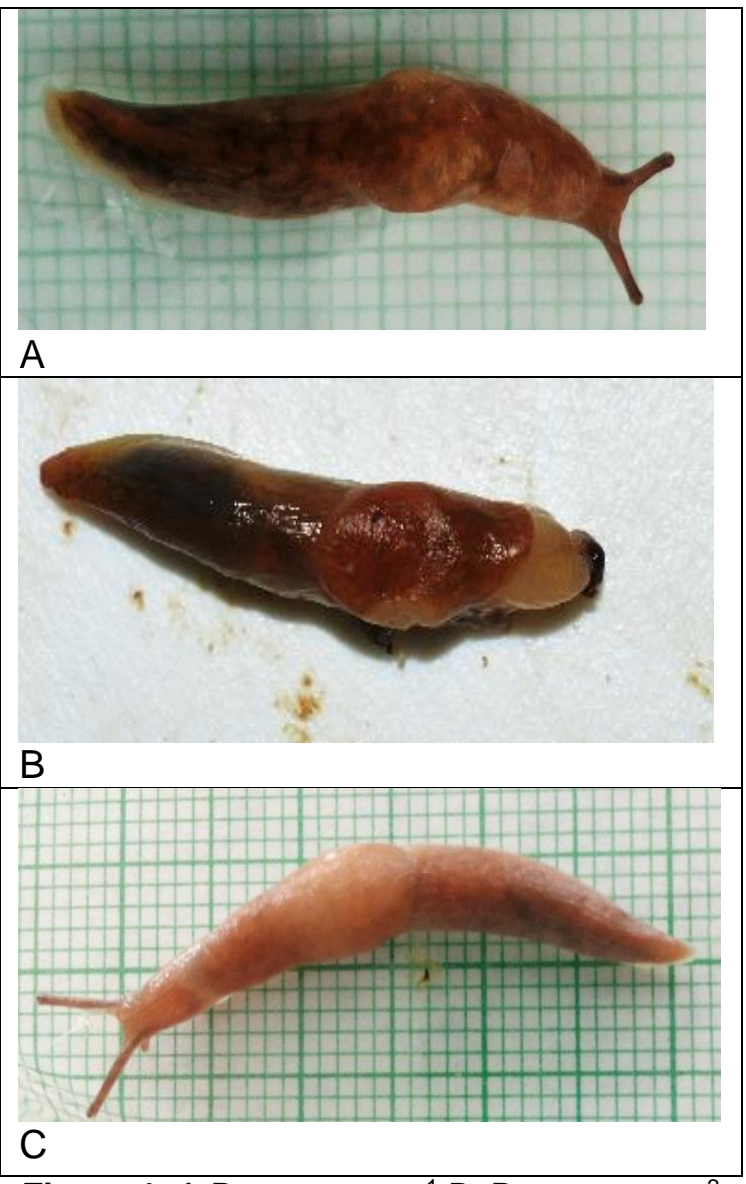

Figura 1. A Deroceras $\mathrm{sp} .{ }^{1} \mathrm{~B}$. Deroceras $\mathrm{sp} .^{2}$ C. Deroceras sp. ${ }^{3}$ Morfoespecies de la Familia Agriolimacidae. Fuente personal, fotografía de especímenes recolectados en campo.

El género Deroceras se encuentra reportado en Colombia para los cultivos de papa negra y criolla (ICA, 2011.b), fresa (SENA y la Secretaría de Agricultura y Desarrollo Rural de Antioquia, 2014), frijol (Arias et al, 2007) y hortalizas en general (ICA, 2012). Sin embargo, solo son mencionadas las especies Deroceras reticulatum Müller, 1774 y Deroceras agreste Linnaeus, 1758, las cuales podrían coincidir con las morfoespecies Deroceras sp. ${ }^{1}$ y Deroceras sp. $^{3}$, respectivamente.

También cabe resaltar la presencia de Deroceras $\mathrm{sp.}^{1}$ en todas las veredas muestreadas de Pamplona, y en todos los cultivos a pesar de no ser reportada en 
algunos de ellos, como la mora y el tomate de árbol.

De la Familia Arionidae se observaron especies pertenecientes a la familia Arionidae, género Arion. La morfoespecie denominada Arion sp. ${ }^{1}$ (Figura 2), presentaba un tamaño de $4,5 \mathrm{~cm}$ aproximadamente, caracterizada por presentar un pneumostoma posicionado en la zona anterior del manto, no contaba con la presencia de quilla, sin embargo, la zona de la quilla era rodeando por una membrana, el extremo posterior del cuerpo era suavemente redondeado y se observaba la presencia de una glándula caudal (con forma de triángulo). Esta se encontró en los nueve cultivos muestreados en Pamplona (arveja, cebolla de bulbo, fresa, frijol, mora, papa criolla, papa negra, tomate de árbol y zanahoria).

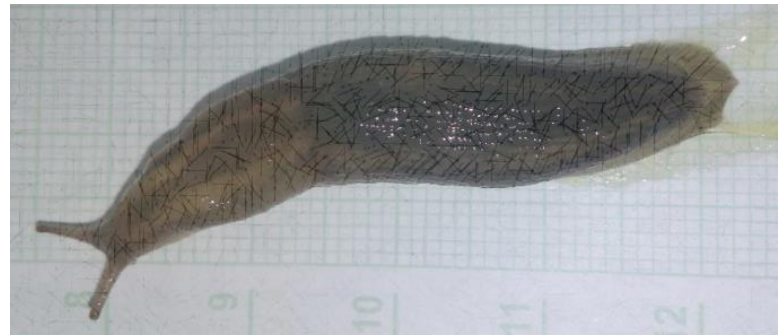

Figura 2. Arion sp. ${ }^{1}$ Morfoespecie de la Familia Arionidae, genero Arion. Fuente personal, fotografía de especímenes recolectados en campo.

Al género al que pertenece la morfoespecie identificada también son atribuidos los daños en distintos tejidos de las plantas (Hernández et al., 2015). Esta especie guarda similitud con las características de Arion subfuscus Draparnaud, 1805, pero su identificación precisa se dificulta ya que pertenece al complejo Arion que agrupa a especies que guardan estrecha similitud (Harwood et al., 2010), demandando una identificación meticulosa y por una persona experta en el tema.

De la Familia Milacidae se encontraron especímenes pertenecientes al género
Milax, la cual se denominó morfoespecie Milax sp. ${ }^{1}$ (Figura 3) presentaba un tamaño de $3,5 \mathrm{~cm}$ aproximadamente, con presencia de quilla con envergadura desde la parte caudal hasta el manto. Su preferencia de cultivos fue fresa, zanahoria y arveja.

La morfoespecie presenta una estrecha similitud con Milax gagates Draparnaud, 1801, de importancia económica para los cultivos de papa (ICA, 2011.b), mora (ICA, 2011.a) y hortalizas (ICA, 2012).

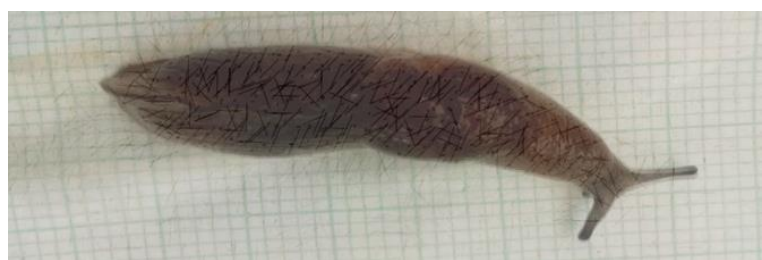

Figura 3. Milax sp. ${ }^{1}$ Morfoespecie de la Familia Milacidae, genero Milax. Fuente personal, fotografía de especímenes recolectados en campo.

La distribución de las morfoespecies encontradas en las veredas muestreadas, independientemente del cultivo en el cual se encontraban fue de la siguiente manera (Tabla 2).

Tabla 2. Distribución de morfoespecies de babosas, por vereda.

\begin{tabular}{|c|c|}
\hline Vereda & Especies \\
\hline Chíchira & Arion sp. ${ }^{1}$, Deroceras sp. ${ }^{1}$, Deroceras sp. ${ }^{2}$ \\
\hline $\begin{array}{l}\text { El } \\
\text { Escorial }\end{array}$ & $\begin{array}{l}\text { Arion sp. }{ }^{1}, \text { Deroceras sp. }{ }^{1}, \text { Deroceras sp. }{ }^{2} \text {, } \\
\text { Deroceras sp. }{ }^{3}\end{array}$ \\
\hline El Rosal & $\begin{array}{l}\text { Milax sp. }{ }^{1}, \text { Arion sp. }{ }^{1}, \text { Deroceras sp. }{ }^{1} \text {, } \\
\text { Deroceras sp. }{ }^{2} \text {, Deroceras sp. }{ }^{3}\end{array}$ \\
\hline $\begin{array}{l}\text { El } \\
\text { Totumo }\end{array}$ & $\begin{array}{l}\text { Milax sp. }{ }^{1}, \text { Arion sp. }{ }^{1} \text {, Deroceras sp. }{ }^{1} \text {, } \\
\text { Deroceras sp. }{ }^{2}, \text { Deroceras sp. }{ }^{3}\end{array}$ \\
\hline $\begin{array}{l}\text { Montead } \\
\text { entro }\end{array}$ & $\begin{array}{l}\text { Milax sp. }{ }^{1}, \text { Arion sp. }{ }^{1} \text {, Deroceras sp. }{ }^{1} \text {, } \\
\text { Deroceras sp. }{ }^{2}, \text { Deroceras sp. }{ }^{3}\end{array}$ \\
\hline
\end{tabular}

Fuente: Autor

Arion estaba presencia en las cinco veredas muestreadas. al igual que Deroceras sp. ${ }^{1}$, Deroceras sp. ${ }^{2}$. Milax sp. ${ }^{1}$ 
estaba solo presente en tres (3) de las cinco (5) veredas visitadas, a pesar de que $M$. gagates está informada como plaga en varios cultivos presentes en las veredas de Pamplona.

\section{Perjuicios causados por las babosas en los cultivos.}

No en todas las veredas se presentaban las mismas especies cultivadas, por lo cual para cada vereda se la incidencia y severidad ponderada por cada cultivo presente (Tabla 3).

Tabla 3. Porcentaje de incidencia y severidad, presente en cada cultivo por vereda.

\begin{tabular}{|c|c|c|c|c|}
\hline Vereda & Cultivo & Campos & Incidencia & Severidad \\
\hline \multirow[t]{5}{*}{ Chíchira } & Arveja & 1 & 48,00 & 9,60 \\
\hline & $\begin{array}{l}\text { Fresa } \\
\text { Papa }\end{array}$ & 3 & 37,00 & - \\
\hline & $\begin{array}{l}\text { criolla } \\
\text { Papa }\end{array}$ & 3 & 68,67 & 15,07 \\
\hline & negra & 2 & 67,00 & 20,80 \\
\hline & $\begin{array}{l}\text { Tomate } \\
\text { de árbol }\end{array}$ & 1 & 30,00 & 6,00 \\
\hline \multirow[t]{3}{*}{ El Rosal } & Arveja & 2 & 53,00 & 12,20 \\
\hline & Fresa & 6 & 30,00 & - \\
\hline & Mora & 2 & 33,00 & 6,60 \\
\hline \multirow{4}{*}{$\begin{array}{l}\text { El } \\
\text { Escorial }\end{array}$} & & & & \\
\hline & $\begin{array}{l}\text { Fresa } \\
\text { Papa }\end{array}$ & 6 & 29,83 & - \\
\hline & criolla & 3 & 53,33 & 12,53 \\
\hline & Zanahoria & 1 & 40,00 & 8,00 \\
\hline \multirow{7}{*}{$\begin{array}{l}\text { El } \\
\text { Totumo }\end{array}$} & & & & \\
\hline & Arveja & 2 & 46,00 & 9,20 \\
\hline & Cebolla & 1 & 42,00 & 8,40 \\
\hline & Fresa & 2 & 39,50 & - \\
\hline & $\begin{array}{l}\text { Frijol } \\
\text { Papa }\end{array}$ & 1 & 26,00 & 5,20 \\
\hline & $\begin{array}{l}\text { criolla } \\
\text { Tomate }\end{array}$ & 2 & 62,00 & 15,40 \\
\hline & de árbol & 2 & 12,00 & 2,40 \\
\hline \multirow{5}{*}{$\begin{array}{l}\text { Monte } \\
\text { Adentro }\end{array}$} & & & & \\
\hline & Arveja & 3 & 72,67 & 16,53 \\
\hline & $\begin{array}{l}\text { Fresa } \\
\text { Pana }\end{array}$ & 4 & 27,00 & - \\
\hline & criolla & 2 & 61,00 & 20,20 \\
\hline & Zanahoria & 1 & 84,00 & 16,80 \\
\hline
\end{tabular}

Fuente: Autor

Para la fresa solo se estimó la incidencia, debido a que el perjuicio ocasionado por las babosas es directamente al fruto comercializable, observándose entre el 25 $\%$ y $40 \%$ de incidencia, valor que representa la proporción de frutos perdidos, encontrandose los valores más altos en las veredas Chíchira y el Totumo (Tabla 4). En el cultivo de fresa (vereda El Rosal) y tomate de árbol (vereda $\mathrm{EI}$ Totumo) se encontraban presentes todas las morfoespecies identificadas. 
Tabla 4. Incidencia y severidad ponderada por cultivo en Pamplona.

\begin{tabular}{lccc} 
Cultivo & Campos & $\begin{array}{c}\text { Incidencia } \\
(\%)\end{array}$ & $\begin{array}{c}\text { Severidad } \\
(\%)\end{array}$ \\
\hline Papa negra & 2 & 67,00 & 20,80 \\
Papa criolla & 10 & 61,25 & 15,80 \\
Zanahoria & 2 & 62,00 & 12,40 \\
Arveja & 8 & 54,92 & 11,88 \\
Cebolla & 1 & 42,00 & 8,40 \\
Mora & 2 & 33,00 & 6,60 \\
Fresa & 21 & 32,67 & - \\
Frijol & 1 & 26,00 & 5,20 \\
Tomate de & & & \\
árbol & 3 & 21,00 & 4,20 \\
\hline Fuente: Autor & & &
\end{tabular}

Fuente: Autor

En cuanto a los demás cultivos el porcentaje de perjuicio se presentaba en relación a al porcentaje de severidad entre el cual los valores más altos (cercanos al $20 \%$ ) se obtuvieron en los cultivos de arveja (Monteadentro), papa criolla (Chíchira, El Totumo y Monteadentro), papa negra (Chíchira) y zanahoria (Monteadentro).

En las veredas que se obtuvieron estos valores más altos se caracterizaban por la presencia de todas las morfoespecies de babosas a excepción de la vereda Chíchira (Tabla 2). Los valores más bajos se evidenciaron en el cultivo de tomate de árbol (Chíchira y el Totumo), estos no superaban ni el $5 \%$ de severidad; a pesar de llegar a realizar perjuicio no son reportadas en la literatura como plagas de importancia para el cultivo.

También los cultivos de cebolla de bulbo, frijol y mora, presentaron bajos niveles de incidencia, sin embargo, la literatura si reporta las babosas como plagas de estos cultivos (ICA, 2012; Arias et al., 2007; ICA, 2011.a).

En Pamplona, los cultivos que presentan los mayores valores de perjuicio ocasionado por babosas fueron la papa negra, papa criolla, la zanahoria y arveja alcanzando entre 50 y $70 \%$ de incidencia y entre el $10 \%$ al $20 \%$ en severidad y entre los menos afectados se encuentran el frijol y el tomate de árbol (Tabla 4).

En la fresa, aunque los valores obtenidos apenas superaron el $30 \%$ de incidencia, es un valor a tener en cuenta por afectar directamente a la producción. Los valores más bajos se encontraron en los cultivos de frijol y tomate de árbol, lo que pudiera atribuirse al control que hacen los productores sobre las babosas en esos cultivos.

\section{CONCLUSIONES}

Se identificaron cinco (5) morfoespecies de babosas afectando los cultivos Pamplona, pertenecientes a las familias Agriolimacidae, Arionidae y Milacidae.

Arion sp. ${ }^{1}$, Deroceras sp. ${ }^{1}$ y Deoceras sp. ${ }^{2}$ estaban presentes en las cinco veredas; Deroceras sp. $^{3}$ no se encontró en la vereda Chíchira mientras que Milax sp. ${ }^{1}$ solo se consiguió en las veredas El Rosal, El Totumo y Monteadentro, prefiriendo todas las morfoespecies al cultivo de fresa y tomate de árbol (veredas El Rosal y El Totumo, respectivamente).

La incidencia de daño por los moluscos se evidencia en los nueve cultivos muestreados bajo las condiciones de Pamplona, sin embargo, la mayor preferencia se observa en papa negra, papa criolla, zanahoria y alverja con los valores más altos de incidencia y severidad, resaltando el nivel de daño que ocasionan las babosas en los frutos del cultivo de fresa.

\section{REFERENCIAS BIBLIOGRAFÍCAS}

Angulo, W. J., Mendoza, J. A. y Uriel, H. U. (2017). Análisis de la vulnerabilidad por fenómenos de remoción en masa en la Cuenca Tanauca estudio de caso. Revista Ambiental Agua, Aire y Suelo. 
ISSN 1900-9178. Volumen (8),

Numero

DOI: https://doi.org/10.24054/1900

9178.v2.n2.2017.3276

ARIAS, J.H., JARAMILLO, M. Y RENGIFO, T. 2007. Manual: Buenas Prácticas Agrícolas, en la Producción de Fríjol Voluble. FAO, Gobernación de Antioquia, MANA, CORPOICA, Centro de Investigación "La Selva".

Camargo, W. C. (2016). Modelación hidrologico-hidraulica de eventos de inundacion en el Rio Bogotá (sector tocanzipa-chia) usando HEC-RAS. Revista Ambiental Agua, Aire y Suelo. ISSN 19009178. Volumen (7), Numero (2). DOI: https://doi.org/10.24054/1900 9178.v2.n2.2016.3267

CAÑEDO, V., ALFARO, A. Y KROSCHEL, J. 2011. Manejo integrado de plagas de insectos en hortalizas. Principios y referencias técnicas para la Sierra Central de Perú. Centro Internacional de la Papa (CIP), Lima, Perú. Disponible en: http://cipotato.org/wpcontent/uploads/2014/08/005739.p df. Consulta 25/10/2018

CIBA GEIGY. 1981. Manual de ensayos de campo en protección vegetal, Basilea. Suiza. 135 p.

CONSTANTINO, L. M., GOMES, S. Y BENAVIDES, P. 2010. Descripción y daños causados por las babosas Colosius pulcher y Sarasinula plebeia en el cultivo de café en Colombia. Centro Nacional de Investigación de Café (Cenicafé). Chinchiná, Caldas, Colombia.

GRIFFITHS, J., PHILLIPS, D. S., COMPTON, S. G., WRIGHT, C. Y INCOLL, L. D. 1998. Responses of slug numbers and slug damage to crops in a silvoarable agroforestry landscape. Ecology and Evolution Group. School of Biology, University of Leeds, Leeds. Journal of Applied Ecology, 35: 252-260.
HARWOOD, J.D., THOMAS, A.K., MC DONNELL, R.J. Y PAINE, T.D. 2010. A Field Guide to the Slugs of Kentucky. University of Kentucky College of Agriculture. Lexington, Kentucky.

HERNÁNDEZ, B., GUERRERO, N. Y SIERRA, M. 2015. Determinación de los daños en babosas (Arion distinctus) causado por la tierra diatomea a diferentes concentraciones bajo condiciones de laboratorio en el ISER. Revista Distancia Al Día. 1:1-9.

HERRERA, N. Y CASTELLANOS, L. 2013. Informe sobre la incidencia de moluscos plaga en organopónicos del municipio de Cienfuegos, Cuba. Centro Agrícola, 40(1): :89-90

HERRERA, N., LÓPEZ, B., CASTELLANOS, L., NODARSE, M. Y PEREZ, Y. 2013. Incidencia de los moluscos plagas en los organopónicos del municipio de Cienfuegos. Centro Agrícola, 40(4); 49-55.

ICA. 2012. Manejo fitosanitario del cultivo de hortalizas. Bogotá D.C. Colombia.

ICA. 2011.a. Manejo fitosanitario del cultivo de la mora (Rubus glaucus Benth). Bogotá D.C. Colombia.

ICA. 2011.b. Manejo fitosanitario del cultivo de la papa (Solanum tuberosum subsp. andigena y $S$. phureja). Bogotá D.C. Colombia.

MARTÍNEZ, J.W., BOHÓRQUEZ, S.L. Y ACOSTA, A. 1994. Determinación taxonómica de cinco grupos de babosas y estudio del ciclo de vida del grupo predominante en un cultivo comercial de alstroemeria de Madrid-Cundinamarca. Agronomía colombiana, XI (1): 5361.

Melo, J., Saavedra, S. y Ramón, J. A. (2017). Evaluación de la adsorcion de CU+2 y azul de metileno en biosorbentes de bajo costo 
obtenidos a partir de biomasa residual de la agroindustria de cítricos. Revista Ambiental Agua, Aire y Suelo. ISSN 1900-9178. Volumen (8), Numero (2). DOI: https://doi.org/10.24054/1900 9178.v2.n2.2017.3277

MÉNDEZ, A.C. Y CASTELLANOS, L. 2017. Eficacia de la tierra de diatomeas contra Helix aspersa en condiciones in vitro en Pamplona, Norte de Santander. Journal of Negative \&

Méndez, A.C., \& Castellanos, L. (2019). Eficacia de la tierra de diatomeas y la cal sobre ariónidos $y$ agriolimácidos. Revista Corpoica. Ciencia Tecnol Agropecuaria, 20(3): 579-593, 2019. 0122-8706, http://revistacta.agrosavia.co/index. php/revista

Meneses, V. B., Álzate, D. y Mosquera, J. (2016). Sistema de optimización de las técnicas de planificación en agricultura de precisión por medio de drones. Revista Ambiental Agua, Aire y Suelo. ISSN 19009178. Volumen (7), Numero (2). DOI: https://doi.org/10.24054/1900 9178.v2.n2.2016.3268

SENA Y SECRETARÍA DE AGRICULTURA $Y$ DESARROLLO RURAL DE ANTIOQUIA. 2014. Manual Técnico del Cultivo de Fresa Bajo Buenas Prácticas Agrícolas. Medellín, Colombia.

SERRE, M. 2005. Manejo de babosas en el cultivo de girasol en siembra directa. Departamento de Agronomía, Pioneer Argentina S.A. Dupon Company. Disponible en: https://www.pioneer.com/CMRoot/l nternational/Argentina Int//AGRON OMIA/con agric inv lotes/IL Mane jobabosa girasol 05.pdf. Consulta 20/07/2018.

Velandia, F. J., Granados, J. D., Ramón, J. D. y Roa, A. L. (2016). Caracterización de consorcios microbianos con potencial degradador de contaminantes en el municipio de Pamplona, Norte de Santander. Revista Ambiental Agua, Aire y Suelo. ISSN 19009178. Volumen (7), Numero (1). DOI: https://doi.org/10.24054/1900 9178.v1.n1.2016.3278

Villamizar, V. A. y Justinico, A. J. (2017). Reconstrucción paleoclimatica y paleoambiental de los territorios de la llanura inundable del araucana a partir del análisis de sedimentos recientes, Departamento de Arauca. Revista Ambiental Agua, Aire y Suelo. ISSN 1900-9178. Volumen (8), Numero DOI: https://doi.org/10.24054/1900 9178.v1.n1.2017.3272 